\title{
Modelagem estatística para estimação da população de protozoários ruminais em função da relação volumoso:concentrado na dieta e da presença de aditivos
}

\author{
Statistical modeling for estimation of rumen protozoa for roughage: concentrate diet \\ and in presence of additives
}

\author{
JESUS, Lisiane Pereira de ${ }^{1^{*}}$; CABRAL, Luciano da Silva ${ }^{1}$; ESPINOSA, Mariano \\ Martinez ${ }^{1}$; ABREU, Joadil Gonçalves de ${ }^{1}$; ZERVOUDAKIS, Joanis Tilemahos ${ }^{1}$; \\ MORENZ, Mirton José Frota ${ }^{2}$
}

\footnotetext{
${ }^{1}$ Universidade Federal de Mato Grosso, Faculdade de Medicina Veterinária, Departamento de Zooetcnia, Cuiabá, Mato Grosso, Brasil.

${ }^{2}$ Universidade Federal Rural do Rio de Janeiro, Seropédica, Rio de Janeiro, Brasil.

*Endereço para correspondência: lisiane.jesus@ig.com.br
}

\section{RESUMO}

Objetivou-se com a realização deste trabalho desenvolver modelos estatísticos para estimar as variações da população de protozoários ruminais em função da relação volumoso:concentrado e da presença ou não de aditivos na dieta. Foram construídas planilhas mediante a utilização do software Excel com dados referentes à composição da dieta, presença e ausência de aditivos e do número de protozoários do rúmen de bovinos de 23 artigos científicos e três teses realizados no Brasil. Estas variáveis foram utilizadas e testadas para obtenção da equação para predição do perfil da população de protozoários. Os modelos estatísticos obtidos foram verificados por análise residual e a seleção das variáveis foi realizada considerando o nível de significância de $5 \%$ de probabilidade do erro experimental. Verificou-se que dietas com maior proporção de volumosos favorecem o incremento na população de protozoários totais e em particular, de Entodinium, enquanto a presença de ionóforos reduz o número desses microorganismos ruminais. $\mathrm{O}$ modelo estatísticos desenvolvido pode auxiliar na identificação antecipada de fatores nutricionais limitantes para o desenvolvimento da microbiota ruminal.

Palavras-chave: ciliados ruminais, microbiota ruminal, ruminantes

\section{SUMMARY}

The objective was to develop statistical models to estimate variations in the population of Protozoa in the light of forage: concentrate ratio and the presence or absence of additives in the diet. Excel spreadsheets with data on diet composition, presence and absence of additives and the number of protozoa in the rumen of cattle for work done in Brazil were using. These variables were used and tested to obtain the equation for predicting the profile of the population of protozoa. The models obtained were checked by residual analysis and selection of variables was performed considering the significance level of $5 \%$. It was found for the total count of protozoa and Entodinium that the increased proportion of large positive effect and additive ionophores had a negative effect. The use of statistical models can provide a better understanding of dietary factors on the microbiota of the rumen and on the animal.

Keywords: rumen ciliates, rumen microbial, ruminants 


\section{INTRODUÇ̃̃̃O}

Embora os protozoários ruminais não sejam a população mais numerosa do rúmen, chegam a representar cerca de metade da biomassa de microorganismos, o que contribui de forma efetiva para os eventos digestivos neste compartimento (DIJKSTRA \& TAMMINGA, 1995). O entendimento de fatores dietéticos e físico-químicos que governam a população de protozoários ruminais tem sido objeto de muitos estudos (KOZLOSKI, 2002). Entretanto, a maioria desses estudos aborda aspectos qualitativos da dieta sobre as populações de protozoários ruminais, porém, sem salientar aspectos quantitativos que determinam o seu aumento ou redução no rúmen.

Jóhannes et al. (2006) observaram acentuada diminuição na população de protozoários na medida em que se incrementa a participação do amido em dietas para ruminantes. Outros autores relataram aumento no número de protozoários à medida que elevaram-se os níveis de concentrado adicionados à dieta (BURGER et al., 2000; HRISTOV et al., 2001; MARTINELE et al., 2008a). Estratégias para modificação do ambiente ruminal são foco de pesquisas (FONSECA \& DIAS-DASILVA, 2001; LOPES et al., 2002; VARGAS et al., 2002; EUGÈNE et al., 2004; PEREIRA et al., 2005; FANDIÑO et al, 2008), também a administração de aditivos alimentares como o uso de ionóforos, que comprovadamente reduzem o número de protozoários (VALINOTE et al., 2005; MARTINELE et al., 2008b; RÍSPOLI et al., 2009). Essa tendência, também tem sido observada no Brasil, uma vez que nos últimos anos houve expressivo aumento no número de publicações que abordaram os efeitos da dieta sobre a população de protozoários ruminais (NOGUEIRA FILHO et al., 2004; GIRI et al., 2005; MIRESAN et al., 2006; MARTINELE et al., 2008a; MARTINELE et al., 2008b). O uso da meta-análise pode contribuir decisivamente para sistematizar as informações disponíveis, bem como integrar e compreender os distintos fenômenos que podem ocorrer no ambiente ruminal (LUIZ, 2002; LOVATTO et al., 2007).

Objetivou-se com a realização este trabalho usar uma abordagem em metaanálise para relacionar dietas de diferentes naturezas com a dinâmica da população de protozoários ruminais.

\section{MATERIAL E MÉTODOS}

As equações de predição foram construidas a partir da seleção de artigos e outras formas de divulgação científica, que relacionavam a concentração de protozoários com descritores dietéticos como a proporção de concentrado e volumoso, teor de fibra em detergente neutro e proteína bruta, e também a presença ou não de ionóforos.

A base de dados utilizada para a realização da meta-análise foi composta por 23 artigos científicos publicados no Brasil (Tabela 1).

A tese, dissertações e dois trabalhos publicados em anais de congresso constam nas referências, pois destes depende a reprodutibilidade do trabalho. Os artigos foram publicados nos seguintes periódicos: Arquivos Brasileiros de Medicina Veterinária e Zootecnia, Ciência e Agrotecnologia, Boletim de Indústria Animal, Pesquisa Agropecuária Brasileira, Revista Brasileira de Zoologia e Revista Brasileira de Zootecnia, no período de 1981 a 2006. 
Rev. Bras. Saúde Prod. Anim., Salvador, v.13, n.1, p...-... jan/mar, 2011 http://www.rbspa.ufba.br ISSN 15199940

Tabela 1. Base de dados utilizada para modelagem estatística conforme ano de publicação (artigos)

\begin{tabular}{|c|c|c|c|c|c|c|}
\hline \multicolumn{7}{|c|}{ Ano de Publicação (1981-1998) } \\
\hline \multicolumn{2}{|l|}{1981} & 1992 & \multicolumn{2}{|c|}{1996} & \multicolumn{2}{|r|}{1998} \\
\hline \multicolumn{2}{|l|}{ Lyle et al. } & Nagajara et al. & \multicolumn{2}{|c|}{ Franzolin \& Dehority } & \multicolumn{2}{|c|}{$\begin{array}{l}\text { D’Agosto \& Santa Rosa } \\
\text { Nogueira Filho et al. }\end{array}$} \\
\hline \multicolumn{7}{|c|}{ Ano de Publicação (2000-2006) } \\
\hline \multicolumn{2}{|l|}{2000} & 2001 & 2002 & \multicolumn{2}{|c|}{2005} & 2006 \\
\hline \multicolumn{2}{|c|}{$\begin{array}{l}\text { Arakiki et al. } \\
\text { Burger et al. } \\
\text { D'Agosto \& Guedes } \\
\text { Franzolin \& Franzolin } \\
\text { Nogueira Filho et al. }\end{array}$} & $\begin{array}{l}\text { Hristov et al. } \\
\text { Sálvio \& } \\
\text { D’Agosto }\end{array}$ & Franzolin et al. & \multicolumn{2}{|c|}{ Valinote et al. } & $\begin{array}{l}\text { Camerini \& Silva } \\
\text { et al. } \\
\text { Rodrigues et al. }\end{array}$ \\
\hline \multicolumn{7}{|c|}{ Ano de Publicação (2007-2009) } \\
\hline $\begin{array}{l}\quad 2007 \\
\text { Martinele et al. } \\
\text { Vidal et al. }\end{array}$ & \multicolumn{2}{|c|}{$\begin{array}{l}\qquad 2008 \\
\text { Martinele et al.(a) } \\
\text { Martinele et al.(b) } \\
\text { Soares et al. }\end{array}$} & $\begin{array}{l}\qquad 2009 \\
\text { Ríspoli et al. } \\
\text { Saran Neto et al. }\end{array}$ & \multicolumn{2}{|c|}{$\begin{array}{c}\text { Tese } \\
\text { Domingues (2006) }\end{array}$} & $\begin{array}{l}\text { Dissertação } \\
\text { Ortolan (2005) } \\
\text { Rivera (2006) }\end{array}$ \\
\hline
\end{tabular}

As informações disponíveis foram sistematizadas em planilhas do software Excel, e foram registradas como variáveis a composição da dieta (relação volumoso:concentrado), presença e ausência de aditivos (monensina), lipídeos e compostos secundários de plantas (taninos), como dados de entrada; perfil da microbiota ruminal, como dados de saída, detalhado em: Protozoários Totais, Família Isotrichidae (Isotricha e Dasitricha), Subfamília Entodiniinae (Entodinium), Subfamília Diplodiniinae (Diplodinium, Eudiplodinium, Metadinium, Eremoplastron, Eodinium, Diploplastron, Elytroplastron, Polyplastron e Ostracodinium) e Subfamília Ophryoscolecinae (Epidinium). Estas variáveis foram utilizadas para obtenção das equações para predição do perfil da microbiota. A verificação da adequação dos modelos foi realizada considerando a análise residual, o valor do coeficiente de determinação $\left(\mathrm{R}^{2}\right)$ e $\quad \mathrm{o} \quad p$-valor dos modelos $(p<0,05)$.

Após verificada a adequação dos modelos, a significância das variáveis foi estabelecida mediante a realização da razão $t$ e seu respectivo valor de $p$, e considerou-se o nível de significância de $5 \%$, através do software MINITAB (Versão 15).

\section{RESULTADOS E DISCUSSÃO}

Independente de gênero ou espécie, podese esperar grande variação no número de micro-organismos ruminais. Parte disso se deve às inúmeras possibilidades de combinação de alimentos presentes na dieta dos ruminantes. Dos estudos consultados pode-se observar que o gênero Entodinium é o principal representante $(80 \%)$ dos protozoários no ambiente ruminal (Tabela 1). Mesmo assim, ao se considerar os valores mínimos e máximos, a amplitude das concentrações relatadas é bastante acentuada, como parte das diferentes naturezas e objetivos dos estudos consultados (Tabela 2). 
Rev. Bras. Saúde Prod. Anim., Salvador, v.13, n.1, p.97-109 jan/mar, 2012 http://www.rbspa.ufba.br ISSN 15199940

Tabela 2. Dados descritivos da concentração de protozoários totais e subpopulações proveniente do rúmen de bovinos (x $10^{4}$ células $/ \mathrm{ml}$ )

\begin{tabular}{lcccccc}
\hline \multicolumn{1}{c}{ Variáveis } & Mínimo & máximo & média & $\begin{array}{r}\% \text { do } \\
\text { total }\end{array}$ & $\mathrm{n}$ & $\begin{array}{r}\text { Desvio } \\
\text { padrão }\end{array}$ \\
\hline Protozoários totais & 6,4 & 114 & 48,01 & 100,00 & 42 & 29,06 \\
Entodinium & 6,01 & 83,90 & 39,75 & 80,00 & 53 & 17,25 \\
Epidinium & 0,5 & 9,96 & 3,87 & 7,80 & 26 & 3,16 \\
Diplodiniinae & 0,10 & 7,00 & 2,55 & 5,10 & 41 & 1,69 \\
Isotrichidae & 0 & 13,0 & 3,51 & 7,10 & 35 & 3,49 \\
\hline
\end{tabular}

A proporção de volumoso pode ser um dos fatores associados à variação no número de protozoários no rúmen (Tabela 3, equação 1). Isso foi devidamente relatado por Kudo et al. (1990), ao observarem que o número médio de protozoários por $\mathrm{ml}^{-1}$ de conteúdo ruminal, foi maior em animais que receberam dietas à base de volumoso (4.6 $\times 10^{4}$ ), quando comparado aos animais que receberam dieta à base de concentrado $\left(8.5 \times 10^{3}\right)$.

Tabela 2. Equações de regressão e valores de $\mathrm{R}^{2}$ das respectivas equações para as populações de protozoarios ruminais

\begin{tabular}{|c|c|c|c|}
\hline Variáveis & $\mathrm{N}^{\mathrm{o}} \mathrm{Eq}$. & Equação & $\mathrm{R}^{2}$ \\
\hline Protozoários Totais & 1 & $\begin{array}{c}(\hat{\mathrm{Y}})=35,0340+0,5471 \text { volumoso }-22,0560 \\
\text { aditivos }-0,0097 \text { vol*con. }\end{array}$ & 0,86 \\
\hline $\begin{array}{l}\text { Protozoários Totais - efeito do } \\
\text { pH ruminal }\end{array}$ & 2 & $\begin{array}{c}\text { Raiz }(\hat{Y})=-17,458+3,3415 \mathrm{pH}-2,2133 \\
\text { aditivos }+0,0011 \text { vol*con }^{*}\end{array}$ & 0,77 \\
\hline Entodinium & 4 & $\begin{aligned} \operatorname{Raiz}(\hat{\mathrm{Y}})= & 5,2756+0,0177 \text { volumoso }-2,3966 \\
& \text { aditivos }+0,0004 \text { vol*con }^{*} \text { con }\end{aligned}$ & 0,67 \\
\hline Epidinium & 5 & $\begin{array}{c}\log (\hat{\mathrm{Y}})=0,4071+0,1754 \text { raiz }\left(\mathrm{x}_{1}\right)+0,1591 \text { raiz } \\
\left(\mathrm{x}_{2}\right)-1,4402 \text { aditivos }\end{array}$ & 0,91 \\
\hline Diplodiniinae & 6 & $\begin{array}{c}\text { Raiz }(\hat{Y})=0,9565+0,0101 \text { volumoso }-0,6175 \\
\text { aditivos }+0,0001 \text { vol*con }^{*}\end{array}$ & 0,73 \\
\hline Isotrichidae & 7 & $\begin{array}{c}\operatorname{Raiz}(\hat{Y})=1,7242+0,0124 \text { volumoso }-1,1876 \\
\text { aditivos }-0,0002 \text { vol*con } .\end{array}$ & 0,70 \\
\hline
\end{tabular}

Na Figura 1 observa-se a estimativa da população de protozoários totais no rúmen a partir da equação (1), em função dos níveis de concentrado na dieta, com a presença ou ausência de aditivos.

A presença de antibióticos ionóforos na dieta dos ruminantes afeta os microorganismos ruminais, independente da relação volumoso:concentrado (Figura 1). Pela natureza da ação desse aditivo (efeito antimicrobiano), é esperada a redução no número de protozoário, como relatado por (ARAKIKI et al., 2000; MACKIE et al., 2002; VALINOTE et al., 2005; MARTINELE et al., 2008b; RÍSPOLI et al., 2009; GOMES et al., 2010). O teor de lipídeos da dieta também está relacionado ao número de ciliados no rúmen (VALINOTE et al., 2005; BALIEIRO-NETO \& MELLOTI, 2007; ALCAIDE \& RUIZ, 2008; MARTINELE et al., 2008b). Elevada participação de concentrados na dieta 
reduz o número total de protozoários, o que pode levar à defaunação ruminal (DIJKSTRA， 1994; FRANZOLIN \& DEHORITY, 1996). O amido na dieta de ruminantes, leva à redução do $\mathrm{pH}$ ruminal, em parte por alterar a salivação e a capacidade de tamponamento no rúmen. Nessas condições há restrições do ambiente ruminal para o desenvolvimento de algumas espécies de microorganismos, aos quais se incluem os protozoários. Burger et al. (2000) observaram comportamento linear decrescente com valores de $9,5 \times 10^{4}$ e 1,4 x $10^{4}$ células/mL, para os níveis de 75 e $90 \%$ de concentrado. Deve ser observado que os protozoários, principalmente os do gênero Entodinium são sensíveis a alteração de $\mathrm{pH}$, utilizam preferencialmente nutrientes insolúveis, como aqueles presentes na parede celular vegetal.

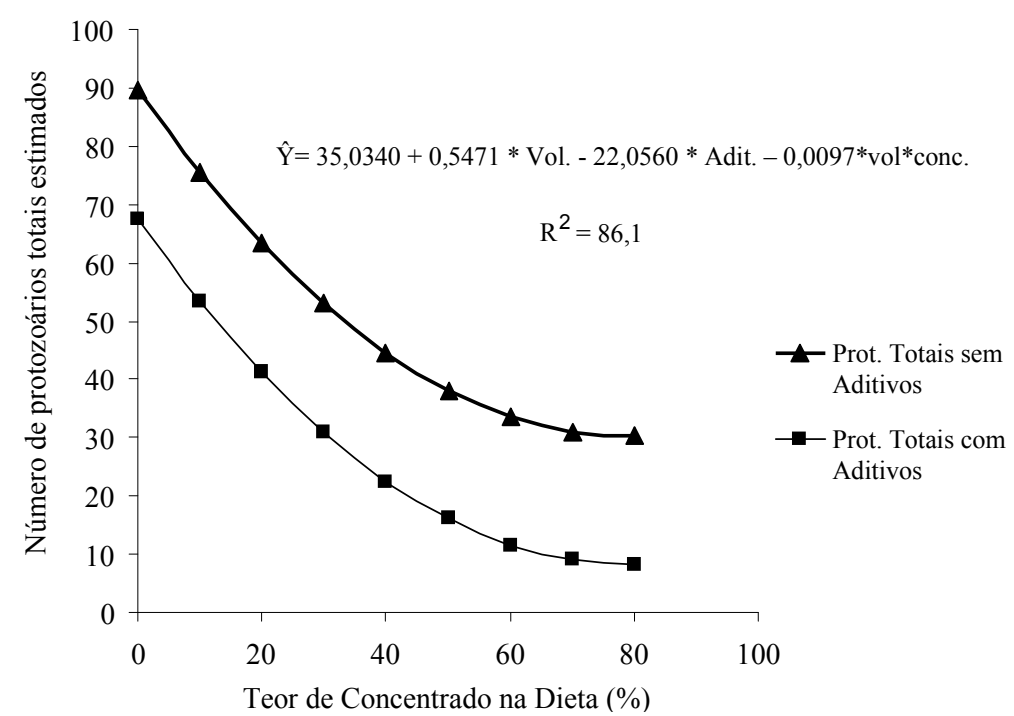

Figura 1. Estimativa do número de protozoários ruminais totais ( $\mathrm{x}$ $10^{4}$ células $/ \mathrm{ml}$ ) em função dos níveis de concentrado na dieta, na ausência ou presença de aditivos

Na Figura 2 são apresentadas estimativas da concentração de protozoários totais no rúmen em função do $\mathrm{pH}$ ruminal, na ausência de aditivos, a partir da equação (2) apresentada na Tabela 2.

$\mathrm{O}$ pH ruminal é função da relação entre a taxa de produção de ácidos graxos voláteis pela microbiota ruminal e os mecanismos tamponantes (secreção salivar, absorção e passagem). Com base na equação e na Figura 2 pode-se perceber que a queda do $\mathrm{pH}$ ruminal abaixo de 6,0 reduzirá a concentração de protozoários totais. Em sistemas de manejo, nos quais esta característica se apresente, é interessante adotar medidas que mantenham o pH acima de 6,0. Como consequência da redução do $\mathrm{pH}$ a permeabilidade da membrana celular, a estabilidade de macromoléculas e atividade de enzimas intra e extracelulares podem ser afetadas. Mesmo assim, micro-organismos tentarão manter o $\mathrm{pH}$ intracelular constante, ou seja, próximo à faixa ótima para seu crescimento. Entretanto, os mecanismos utilizados pelos micro-organismos para a manutenção do $\mathrm{pH}$ intracelular são consumidores de energia e, desta forma, 
podem causar redução do seu crescimento (MADIGAN \& MARTINKO, 2006).

De acordo com Jouany (1996), a redução da população de protozoários pode aumentar a eficiência de síntese de proteína microbiana ( $\mathrm{N}$ microbiano formado $/ \mathrm{kg}$ matéria orgânica fermentada) de 40 a $125 \%$, e o fluxo de proteína microbiana para o abomaso em $47 \%$ (valor médio). Segundo estimativas do National Research Council (NRC, 2001) cada $1 \mathrm{~kg}$ de nutriente digestível total (NDT) consumido permite o fluxo de $130 \mathrm{~g}$ de proteína microbiana para o abomaso. Nesse caso a defaunação poderia aumentar esse valor para $189,8 \mathrm{~g} / \mathrm{kg}$ de NDT (Figura 3).

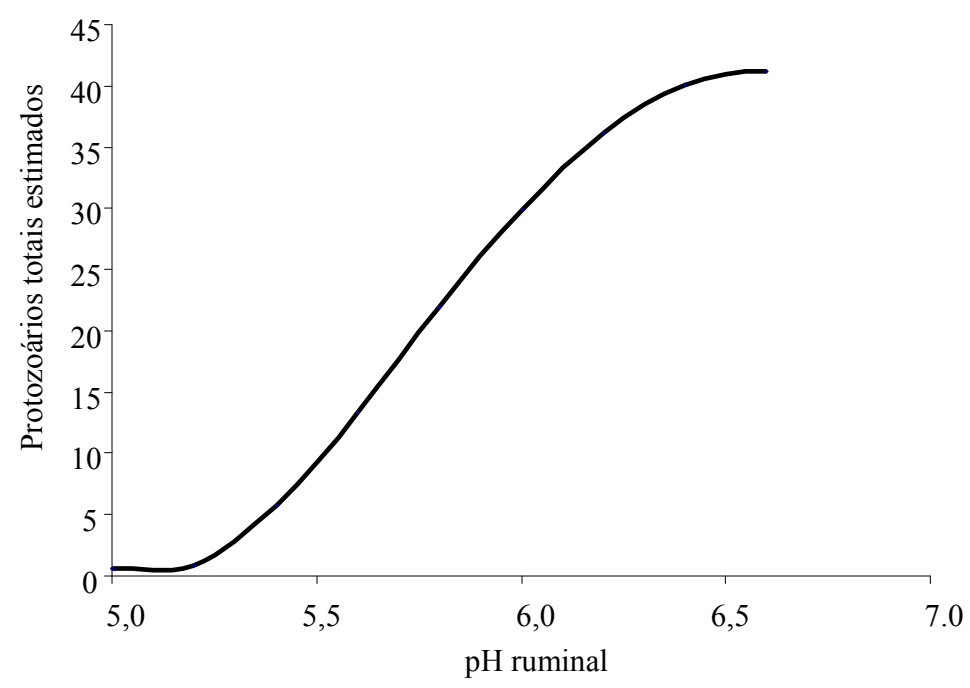

Figura 2. Estimativa do número de protozoários totais no rúmen $\left(\mathrm{x} 10^{4}\right.$ células $\left./ \mathrm{ml}\right)$ em função da variação do $\mathrm{pH}$ ruminal (sem aditivos)

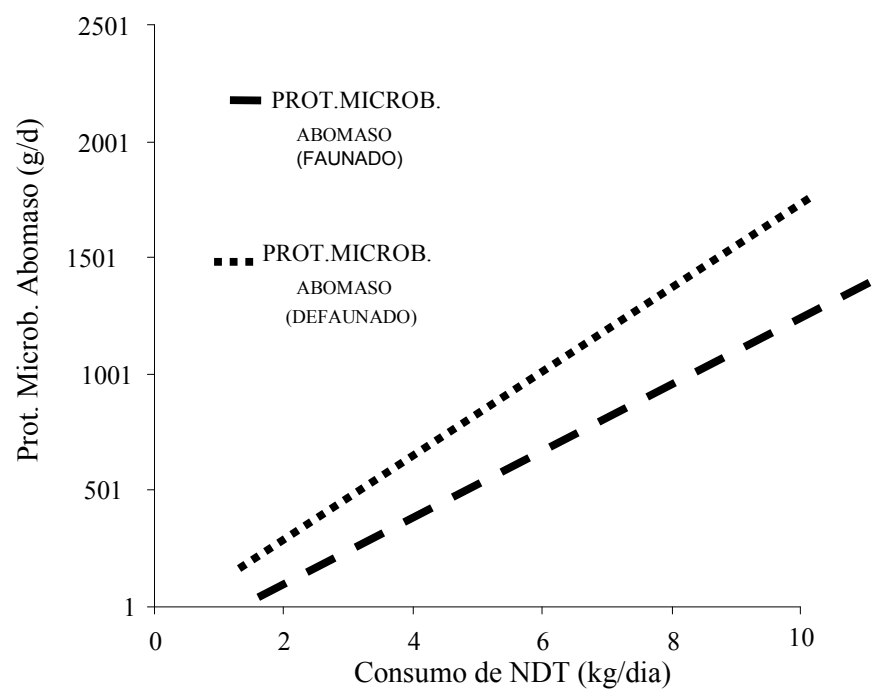

Figura 3. Estimativa do aporte de proteína microbiana em função do níveis consumo de NDT $(\mathrm{kg} / \mathrm{dia})$ em animais faunados e defaunados 
Muitas alterações no ambiente ruminal podem alterar a dinâmica ruminal o que dificulta o processo de predição do desempenho animal a partir dos componentes dietéticos, particularmente quando modelos estáticos são utilizados na predição (DIJKSTRA, 1994).

Neste estudo, o número médio total de ciliados por $\mathrm{mL}$ de conteúdo em dietas sem aditivos e em dietas que utilizaram algum tipo de aditivo (monensina, óleo ou tanino) foi de 58,09 x $10^{4}$ células $/ \mathrm{ml}$ e $27,86 \times 10^{4}$ células $/ \mathrm{ml}$, respectivamente. A presença de aditivos promoveu redução na população total de protozoários, portanto como comprovado pelo modelo estatístico, a presença de aditivos reduz as populações de ciliados, comportamento esse verificado por Arakiki et al. (2000), Valinote et al. (2005), Martinele et al. (2008b), Ríspoli et al. (2009) e Gomes et al. (2010).

$\mathrm{Na}$ Tabela 2 observa-se a equação de regressão ajustada para o número de protozoários do gênero Entodinium (x $10^{4}$ células $/ \mathrm{ml}$ ). À medida que aumenta a proporção de volumoso na dieta, aumenta o número de protozoários do gênero Entodinium. Este resultado pode ser explicado porque o gênero Entodinium, compõem mais de $70 \%$ da população total de protozoários ruminais, que proliferam em dietas à base de volumoso (OGIMOTO \& IMAI, 1981).

A predominância de ciliados da Subfamília Diplodiniinae no rúmen de bovinos, especialmente de organismos do gênero Entodinium, conforme Siqueira \& D'Agosto (2003) foi confirmada neste estudo, o qual registrou em média, $80 \%$ da população total de protozoários composta por estes micro-organismos. Franzolin \& Franzolin (2000) afirmam que as espécies deste gênero compreendem $79,2 \%$ da composição da fauna de bovinos. A predominância do gênero Entodinium foi igualmente verificada em bovinos por Manella \&
Lourenço (2004) e Martinele et al. (2007) que obtiveram, respectivamente $74,8 \%$ e $78,7 \%$ da população total de ciliados, valores bem próximos aos determinados neste estudo (Tabela 1). Esta população representa uma grande parcela dos protozoários totais e, portanto, sofrem da mesma forma $o$ efeito negativo dos aditivos, uma vez que a população é reduzida em função da adição de ionóforo à dieta. Arakiki et al. (2000), ao avaliarem os efeitos da monensina sobre a população de protozoários observaram diminuição na percentagem de Entodinium em animais que recebiam dietas com adição do ionóforo. A interação volumoso $\mathrm{x}$ concentrado foi utilizada nesta equação para fins de ajuste.

Na Tabela 2 observa-se a equação de regressão ajustada para o número de protozoários do gênero Epidinium (x $10^{4}$ células $/ \mathrm{mL}$ ). $\mathrm{O}$ número de protozoários do gênero Epidinium aumenta à medida que diferentes doses de volumoso e concentrado são administradas na dieta. Deve ser observado que Epidinium sp. são ativos na degradação tanto de amido (concentrado), quanto de celulose/hemicelulose (volumoso), (BAILEY \& HOWARD, 1963).

A presença de aditivos reduziu significativamente as populações de Epidinium, o que demonstra efeito similar àquele constatado para $\mathrm{o}$ número de protozoários totais e Entodinium. Martinele et al. (2008b) ao estudarem os efeitos da adição de monensina à dieta observaram a diminuição na contagem de Epidinium quando adicionado o ionóforo. Na Tabela 2 observa-se a equação de regressão ajustada para o número de protozoários pertencentes à SubFamília Diplodiniinae (x $10^{4}$ células $\left./ \mathrm{ml}\right)$. Para Diplodiniinae, com relação à variável volumoso, os resultados foram semelhantes aos obtidos para Entodinium, o que demonstra que estes dois grupos de 
protozoários se comportam da mesma forma mediante alterações na dieta. Esta mesma população foi afetada negativamente pela presença de aditivos, o que evidencia comportamento similar àquele observado para protozoários totais, Entodinium e Epidinium. Da mesma forma, Ríspoli et al. (2009) observaram efeito redutor do tratamento com monensina sobre ciliados da Subfamília Diplodiniinae. A interação volumoso e concentrado foi utilizada como variável de ajuste.
Na Tabela 2 observa-se a equação de regressão ajustada para o número de protozoários da Família Isotrichidae (x $10^{4}$ células $\left./ \mathrm{ml}\right)$. A interação volumoso e concentrado embora sem efeito significativo foi mantida na equação de regressão apenas para efeito de ajuste.

Na Figura 4 observa-se a estimativa do número de protozoários da Família Isotrichidae em função dos níveis de concentrado e da presença ou ausência de aditivos.

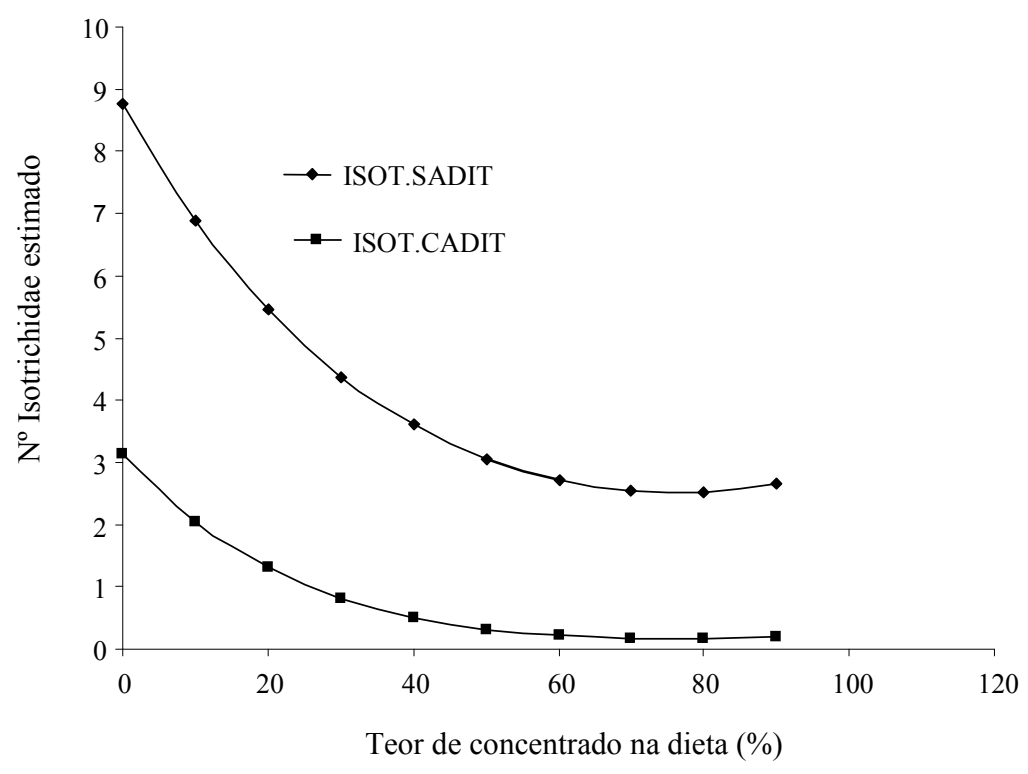

Figura 4. Estimativa do número de protozoários da Família Isotrichidae (x $10^{4}$ células $/ \mathrm{ml}$ ) em função dos níveis de concentrado na dieta, na ausência (SADIT) ou presença (CADIT) de aditivos

Cabe resaltar que esta população prolifera intensamente em dietas à base de concentrado, mas sua representatividade (cerca de 5\%) ainda é muito pequena quando comparada ao primeiro grupo.

Contrariamente, a variável volumoso mostrou efeito significativo, uma vez que houve aumento na população de protozoários da Familía Isotrichidae (Tabela 1), isto porque holotríquios estão presentes em maior número quando a dieta é rica em concentrado, (OGIMOTO \& IMAI, 1981). No entanto, as enzimas responsáveis pela degradação de celulose e hemicelulose têm sido reportadas em protozoários holotríquios, embora em níveis baixos quando comparados com os protozoários entodiniomorfos (WILLIAMS \& COLEMAN, 1991).

Martinele et al. (2008b) observaram que a adição de óleo de soja à dieta reduziu o número de protozoários Isotricha $\mathrm{e}$ 
Dasytricha; a adição de monensina diminui o número de protozoários do gênero Dasytricha, o que confirma o efeito negativo dos aditivos sobre Isotrichidae.

O uso de aditivos comprovadamente reduz as populações de ciliados, fato evidenciado para todas variáveis em estudo. De posse desta informação o pesquisador pode manipular o ambiente ruminal mediante alterações nas proporções de aditivos utilizadas na dieta. Volumosos, concentrados e aditivos afetam distintamente a população de protozoários no rumen. Enquanto a maior presença de volumosos na dieta favorece ao incremento na população de protozoários ruminais, concentrados $\mathrm{e}$ aditivos (monensina, taninos e lipídeos) agem como inibidores do crescimento desses micro-organismos.

Reduzir a quantidade de protozoários no rúmen pode ter consequências sérias na digestão da fibra e na disponibilidade de nutrientes, como a proteína sintetizada no rúmen por estes organismos. $\mathrm{Na}$ formulação de dietas para ruminantes, deve-se levar em consideração a melhor combinação de componentes que resultem na máxima produção microbiana. Nesse sentido, pode ser de extrema utilidade a disponibilidade de modelos preditivos que permitam avaliar os efeitos da dieta na população microbiana.

\section{REFERÊNCIAS}

ALCAIDE, E.M.; RUIZ, D.R.Y. Potencial use of olive by-products in ruminant feeding: a review. Animal Feed Science and Technology, v.147, n.1, p.247-264, 2008.
ARAKAKI, L.C.; STAHRINGER, R.C.; GARRETT, J.E.; DEHORITY, B.A. The effects of feeding monensin and yeast culture, alone or in combination, on the concentration and generic composition of rumen protozoa in steers fed on low-quality pasture supplemented with increasing levels of concentrate. Animal Feed Science and Technology, v.84, n.1-2, p.121-127, 2000 .

BAILEY, R.W.; HOWARD, B.H. Carbohydrases of the rumen ciliate Epidinium eucaudatum (Crawley). Biochemical Journal, v.87, p.146-151, 1963.

BALIEIRO NETO, G.; MELLOTI, L. Produção de ácidos graxos voláteis e contagem deprotozoários ruminais em bovinos suplementados com gordura.

Brazilian Journal of Veterinary Research and Animal Science, v.44, n.2, p.115-121, 2007.

BURGER, P.J., PEREIRA, J.C.; VALADARES FILHO S.C.; SILVA, J.F.C.; QUEIROZ, A.C.; CECON, P.R.; MAGIERO, D. Fermentação ruminal e eficiência microbiana em Bezerros Holandeses Alimentados com dietas contendo diferentes níveis de concentrado. Revista Brasileira de Zootecnia, v.29, n.1, p.215-224, 2000.

CAMERINI E SILVA, R.A.; ARCURI, P.B. D'AGOSTO, M.T.; ODENYO, A.A.; LOPES, F.C.F.; RIBEIRO, M.T.; PEREIRA, L.Z.; DORNELAS, R.A.C. Perfil das populações de protozoários ciliados do rúmen de vacas nãolactantes recebendo dietas ricas em taninos. In: SEMANA DE BIOLOGIA, 29 e MOSTRA DE PRODUÇÃO CIENTÍFICA, 12, Juiz de Fora, MG. Resumos... Juiz de Fora: Universidade Federal de Juiz de Fora, 2006. 
D'AGOSTO, M.; GUEDES, P.M.M.

Caracterização das populações de ciliados do rumem de bovinos de corte no estado de Minas Gerais, Brasil. Revista

Brasileira de Zoociências, v.2, n.1, p.81$90,2000$.

D'AGOSTO, M.; SANTA-ROSA, M.R. Influência do hospedeiro no perfil populacional e nas populações de cillados do rúmen de bovinos. Revista Brasileira de Zoologia, v.15, n.2, p.389-396, 1998.

DIJKSTRA, J. Simulation of the dynamics of protozoa in the rumen. British Journal of Nutrition, v.72, p.679-699, 1994.

DIJKSTRA, J.; TAMMINGA, S. Simulation of the effects of diet on the contribution of rumen protozoa to degradation of fibre in the rumen. British Journal of Nutrition, v.74, p.617-634, 1995.

DOMINGUES, J.L. Avaliação do desempenho em confinamento do metabolismo ruminal e do perfil de ácidos graxos da carne em bovinos nelore, utilizando milho com alto teor de óleo nas dietas de terminação. 2006. 101p. Tese (Doutorado) - Faculdade de Zootecnia e Engenharia de Alimentos, Universidade de São Paulo, Pirassununga.

EUGÈNE, M.; ARCHIMÈDE, H.; MICHALET-DOREAU, B.; FONTY, G. Effects of defaunation on microbial activities in the rumen of rams consuming a mixed diet (fresh Digitaria decumbens grass and concentrate). Animal Reserach, v.53, n.3, p.187-200, 2004.

FANDIÑO, I.; CALSAMIGLIA, S.; FERRET, A.; BLANCH, M. Anise and capsicum as alternatives to monensin to modify rumen fermentation in beef heifers fed a high concentrate diet. Animal Feed Science and Technology, v.145, n.1, p.409-417, 2008.
FONSECA, A.J.M.; DIAS-DA-SILVA, A.A. Efeitos da eliminação dos protozoários do rumem no desempenho produtivo de ruminantes - Revisão.

Revista Brasileira de Ciência Veterinária, v.96, n.538, p. 60-64, 2001.

FRANZOLIN, R.; DEHORITY, B.A. Effect of prolonged high-concentrate feeding on ruminal protozoa concentrations. Journal of Animal Science, v.74, n.11, p.2803-2809, 1996.

FRANZOLIN, R., FRANZOLIN, M.H.T. População de Protozoários Ciliados e Degradabilidade Ruminal em Búfalos e Bovinos Zebuínos sob dieta à base de cana-de-açúcar. Revista Brasileira de Zootecnia, Viçosa v. 29, n.6, p.18531861, 2000.

FRANZOLIN, M.H.T.; SILVEIRA, A.C.; FRANZOLIN, R. Efeitos de dietas com diferentes níveis de fibra em detergente neutro e do tamanho de poros de sacos de náilon incubados no rúmen sobre a fauna ruminal em bubalinos e bovinos. Revista Brasileira de Zootecnia, v.31, n.2, p.716-723, 2002.

GIRI, S.S.; JAGGI, S.; PATHAK, N.N. Feeding of grainless diets containing different nitrogen sources to crossbred growing bulls: effects on rumen fermentation pattern, microbial enzyme activity and ciliate protozoa population. Animal Feed Science and Technology, v.118, n.3-4, p.187-200, 2005.

GOMES, R.C.; ANTUNES, M.T.; NOGUEIRA FILHO, J.C.M.; ÍTAVO, L.C.V.; LEME, P.R. Leveduras vivas monensina em dietas de alto concentrado para bovinos: parâmetros ruminais e degradabilidade "in situ".

Revista Brasileira de Saúde e Produção Animal [Online], v.11, n.1, p.202-216, 2010. 
HRISTOV, A.N.; IVAN, M.; RODE, L.M.; MCALLISTER, T.A.

Fermentation characteristics and ruminal ciliate protozoal populations in cattle fed medium- or high-concentrate barley-based diets. Journal of Animal Science, v.79, p.515-524, 2001.

JÓHANNES, S.; MURPHY,M.; UDÉN, P. Effect of the level of dry matter and protein and degradation rate of starch on in vitro ruminal fermentation. Animal Feed Science and Technology, v.130, n.3-4, p.191-203, 2006.

JOUANY, J.P. Effects of rumen protozoa on nitrogen metabolism by ruminants. Journal of Nutrition, v.126, p.1335S-1346S, 1996.

\section{KOZLOSKI, G.V. Bioquímica dos} ruminantes. Santa Maria: Universidade Federal de Santa Maria, 2002. 140p.

KUDO, H.; CHENG, K.J.; IMAI, S.; HAN, S.S.; COSTERTON, J.W. Effects of feed on the composition of the rúmen ciliate protozoal population in cattle and its relationship to cellulolytic ciliate protozoa. Animal Feed Science and Technology, v.29, n.1-2, p.159-169, 1990.

LOPES, F.C.F.; AROEIRA, L.J.M.; ARCURI, P.B.; DAYRELL, M.S.; VITTORI, A. Efeitos da defaunação em ovinos alimentados com cana-deaçúcar (Saccharum officinarum, L.) adicionada de uréia. Arquivo Brasileiro de Medicina Veterinária e Zootecnia, v.54, n.2, p.180-188, 2002.

LOVATTO P.A.; LEHNEN C.R.; ANDRETTA I.; CARVALHO A.D.; HAUSCHILD L. Meta-análise em pesquisas científicas - enfoque em metodologias. Revista Brasileira de Zootecnia, v.36, p.285-294, 2007. Supl.
LUIZ, A.J.B. Meta-análise: definição, aplicações e sinergia com dados espaciais. Cadernos de Ciência e Tecnologia, v.19, n.3, p.407-428, 2002.

LYLE, R.R.; JOHNSON, R.R.; WILHITE, J.V.; BACKUS, W.R. Ruminal characteristics in steers as affected by adaptation from forage to all concentrate diets. Journal of Animal Science, v.53, n.5, p.1383-1390, 1981.

MACKIE, R.I.; McSWEENEY,C.S.; KLIEVE, A.V. Microbial ecology of the ovine rumen. In: FREER, M.; DOVE, H. (Eds). Sheep Nutrition. Wallingford: CAB International, 2002, 377p.

MADIGAN, M.T.; MARTINKO, J.M. Brock biology of microorganisms. 11.ed. New Jersey: Pearson, 2006, 992p.

MANELLA, M.Q.; LOURENÇO, A.J. População de protozoários ciliados no rúmen de bovinos nelore em pastos de Brachiaria brizantha marandu recebendo suplemento protéico ou com livre acesso a banco de proteína de Leucena leucocephala nas diferentes estações do ano. Boletim da Indústria Animal, n.1, p.1-11, 2004.

MARTINELE, I.; DETONI, M.L.; RUST, M.N.; D'AGOSTO, M. Protozoários ciliados (Protista, Ciliophora) no conteúdo do rúmen e retículo de bovinos. Revista Brasileira de Zooctecnia, v.9, n.1, p.63-66, 2007.

MARTINELE, I.; SIQUEIRACASTRO, I.C.V.; D'AGOSTO, M. Protozoários ciliados no rúmen de bovinos alimentados com dietas de capim-elefante e com dois níveis de concentrado. Revista Brasileira de Saúde e Produção Animal [Online], v.9, n.1, p.74-81, 2008 a. 
MARTINELE, I.; EIFERT, E.C.; LANA, R.P.; ARCURI, P.B.; D'AGOSTO, M. Efeito da monensina e do óleo de soja sobre os protozoários ciliados do rúmen e correlação dos protozoários com parâmetros da fermentação ruminal e digestivos. Revista Brasileira de Zootecnia, v.37, n.6, p.1129-1136, $2008 b$.

MINITAB 15, Software Estatístico, versão 15.1.3., tamanho $96 \mathrm{MB}$, sistema XP, Empresa lidersoftwares, 2010.

MIRESAN, V.; RADUCU, C.; STETCA, G.H. The efect of ruminal defaunation in establisghing the role of the infusores in ruminal physiology. Animal of Science Biotechnology, v.63, p.88-92, 2006.

NAGARAJA, T.G.; TOWNE, G.; BEHARKA, A.A. Moderation of Ruminal Fermentation by Ciliated Protozoa in Cattle Fed a High-Grain Diet. Applied Environmental Microbiology, v.58, n.8, p.2410-2414,1992.

NATIONAL RESEARCH COUNCIL NRC. Nutrient requeriments of dairy cattle. 7.ed. rev. Washinton, D.C., 2001. $381 p$.

NOGUEIRA FILHO, J.C.M.; OLIVEIRA, M.E.M.; TOLEDO, L.R.A.; VELLOSO, L. Protozoários ciliados no rúmen de zebuínos e bubalinos submetidos a dietas com volumosos e concentrados. Revista Pesquisa Agropecuária Brasileira, v.33, n.6, p.993-999, 1998.

NOGUEIRA FILHO, J.C.M.; OLIVEIRA, M.E.M.; ABLAS, D.S.; TITTO, E.A.L.; TOLEDO, L.R.A.; OLIVEIRA, T.S.B.M. Fauna ciliada do rúmen de zebuínos e bubalinos em Pirassununga, São Paulo, Sudeste do Brasil. Acta Scientiarum, v.22, n.3, p.663-668, 2000.
NOGUEIRA FILHO, J.C.M.; OLIVEIRA, M.E.M.; CUNHA, J.A., TOLEDO, L.R.A. Volume líquido e taxa de turnover no rúmen de zebuínos e bubalinos submetidos a dietas com volumosos e concentrados e sua relação com protozoários ciliados. Ciência Animal Brasileira, v.5, n.1, p.1-7, 2004.

OGIMOTO, K.; IMAI, S. Atlhas of rumen microbiology. Tokyo: Japan Scientific Societies Press, 1981. 231p.

ORTOLAN, J.H. Efeitos da levedura, monensina sódica e salinomicina na degradabilidade, digestibilidade, parâmetros ruminais e protozoários ciliados de novilhos Nelore arraçoados com dietas concentradas. 2005. 61p. Dissertação (Mestrado) - Faculdade de Zootecnia e Engenharia de Alimentos, Universidade de São Paulo, Pirassununga.

PEREIRA, J.C.; RIBEIRO, M.D.; VIEIRA, R.A.M.; PACHECO, B.M. Avaliação de modelos matemáticos para o estudo da cinética de passagem de partículas e de fluídos por bovinos em pastagem recebendo suplementos contendo diferentes níveis de proteína não degradável no rúmen. Revista Brasileira de Zootecnia, v.34, n.6, p.2475-2485, 2005.

RÍSPOLI, T.B.; RODRIGUES, I.L.; MARTINS NETO, R.G.; KAZAMA, R.; PRADO, O.P.P.P.; ZEOULA, L.M.; ARCURI, P.B. Protozoários ciliados do rúmen de bovinos e bubalinos alimentados com dietas suplementadas com monensina ou própolis. Pesquisa Agropecuária Brasileira, v.44, n.1, p.92-97, 2009. 
]RIVERA, A.R. Estudo da fermentação ruminal por bovinos consumindo feno de tifton 85 e concentrado com aditivos. $2006.57 \mathrm{p}$. Dissertação (Mestrado em Zootecnia) Faculdade de Ciências Agrárias e Veterinárias, Universidade Estadual de São Paulo, Jaboticabal.

RODRIGUES, I.L; MARTINELE, I.; DETONI, M.L. Perfil de comunidade de protozoários ciliados (Protista, Ciliophora) no rúmen e no retículo de bovinos. In: SEMANA DE BIOLOGIA, 29 e MOSTRA DE PRODUÇÃO CIENTÍFICA, 12, Juiz de Fora, MG. Resumos... Juiz de Fora: Universidade Federal de Juiz de Fora, 2006.

SÁLVIO, G.M.M., D'AGOSTO, M. Ciliados nas cavidades do estômago dos bovinos. Arquivo Brasileiro de Medicina Veterinária e Zootecnia, v.53, n.6, p.686-690, 2001.

SARAN NETTO, A.; ZANETTI, M.A.; PAIVA, F.A.; SALLES, M.S.V.; DEL CLARO, G.R.; LOPES, L.C.; NOGUEIRA FILHO, J.C.M. Efeitos da fonte de enxofre sobre a população de protozoários e degradabilidade no rúmen. Arquivo Brasileiro de Medicina Veterinária e Zootecnia, v.61, n.4, p.910-917, 2009.

SIQUEIRA, I.C.V.; D'AGOSTO, M. Comportamento e perfil de comunidade de protozoários ciliados no rúmen de bovinos. Revista Brasileira de

Zootecnia, v.5, n.2, p.243-252, 2003.
SOARES, P.C.; MARTINELE, M.D.; MARUTA, C.A.; SUCUPIRA, M.C.A.; ANTONELLI, A.C.; MORI, C.S.; ORTOLANI, E.L. Effect of an energydeficient diet on populations of ciliate protozoans in bovine rumen. Arquivo Brasileira de Medicina Veterinária e Zootecnia, v.60, n.1, p.148-155, 2008.

VALINOTE, A.C.; NOGUEIRA FILHO, J.C.M.; LEME, P.R.; LUZ E SILVA, S.; CUNHA, J.A. Fontes de lipídeos e monensina na alimentação de novilhos Nelore e sua relação com a população de protozoários Ciliados do rúmen. Revista Brasileira de

Zootecnia, v.34, n.4, p.1418-1423, 2005.

VARGAS, L.H.; LANA, R.P.; JHAM, G.N.; SANTOS, F.L.; QUEIROZ, A.C.; MANCIO, A.B. Adição de lipídios na ração de vacas leiteiras: parâmetros fermentativos ruminais, produção e composição do leite. Revista Brasileira de Zootecnia, v.31, n.1, p.522-529, 2002. Supl.

VIDAL, J.M.; PAIVA, P.C.A.; ARCURI, P.B., LOPES, F.C.F.; ZAQUINI, L. Efeito de diferentes doses de enxofre no consumo voluntário e nas populações de protozoários do rúmen de novilhas mestiças alimentadas com capim-elefante de baixa qualidade.

Ciência e Agrotecnologia, v.31, n.1, p.218-222, 2007.

WILLIAMS, A.G.; COLEMAN, G.S. The rumen protozoa. London: Blackie Academic, 1991, 423p.

Data de recebimento: 03/08/2011

Data de aprovação: 09/02/2012 